\title{
Expediting Protein Structural Analysis with an Efficient Kernel Density Estimation Algorithm
}

\author{
Yen-Jen Oyang*, Darby Tien-Hau Chang, Chien-Yu Chen, and Shien-Ching Hwang \\ Department of Computer Science and Information Engineering, \\ National Taiwan University, Taipei, Taiwan, R. O. C.
}

yjoyang@csie.ntu.edu.tw, \{darby,cychen,schwang\}@mars.csie.ntu.edu.tw

\begin{abstract}
This paper describes a kernel density estimation based mechanism aimed at expediting protein structural analysis. This study has been motivated by the observation that many protein structural analysis algorithms suffer high time complexity, while only the residues and atoms on the contour of a protein are essential for determining the functions of the protein and how it could interact with the other proteins. Accordingly, for some protein structural analysis problems, it is desirable to invoke a mechanism that can extract the residues and atoms on the contour of a protein in order to expedite the analysis process. The conventional approach to carry out this task is to invoke the $\alpha$-hull algorithm from computer graphics, which features $O\left(n^{2}\right)$ time complexity, where $n$ is the number of residues or atoms in the protein. In this paper, a kernel density estimation based expediting mechanism with an average time complexity of $O(n \log n)$ is proposed. This paper also reports the experiment conducted to evaluate the effects of applying the proposed expediting mechanism to a real protein structural analysis problem. Experimental results reveal that a speedup of 4.8 to 10.3 times can be achieved with minimum impact on the analysis accuracy.
\end{abstract}

\section{Keywords}

protein structural analysis, kernel density estimation, protein tertiary structure.

\section{Introduction}

In recent years, analysis of the tertiary structures of proteins has attracted a high degree of interest in the bioinformatics research community [7,9]. The main reason is that the information obtained from structural analysis of a protein is essential for determining its functions and how it could interact with other proteins. However, many modern protein structural analysis algorithms suffer high time complexity. For example, if the geometric hashing algorithm proposed in [8] is applied to carry out general protein structural alignment, then the time complexity of the process is
$\mathrm{O}\left(n_{1}^{3} n_{2}^{3}\left(n_{1}+n_{2}\right)\right)$, where $n_{1}$ and $n_{2}$ are the numbers of atoms in the two proteins, respectively. Even with some biochemistry knowledge incorporated to narrow down the search domain, the time complexity of the geometric hashing based alignment algorithm proposed in [13] is still $\mathrm{O}\left(n_{1} n_{2}\left(n_{1}+n_{2}\right)\right)$, where $n_{1}$ and $n_{2}$ are the numbers of residues in the two proteins, respectively. Therefore, it is of great significance to develop expediting mechanisms.

One basic observation of protein structural analysis is that only the residues and atoms on the contour of a protein are essential for determining the functions of the protein and how it could interact with the other proteins. Therefore, one possible approach to expedite the analysis process is to exclude those residues and atoms that are not on the contour of the protein. To achieve this goal, we must be able to distinguish those residues and atoms that are on the contour of the protein from those that are not. One conventional approach to tackle this problem is to invoke the $\alpha$-hull algorithm in computer graphics [4]. However, the time complexity of the $\alpha$-hull algorithm is still $\mathrm{O}\left(n^{2}\right)$, where $n$ is the number of atoms or residues that the protein contains. In this paper, an efficient mechanism based on a novel kernel density estimation algorithm is proposed. The time complexity of the proposed expediting mechanism is $\mathrm{O}(n \log n)$. In fact, kernel density estimation is a problem that has been extensively studied by statistician [12]. The main distinction of the novel kernel density estimation algorithm employed in this paper is its low time complexity, $\mathrm{O}(n \log n)$.

This paper also reports the experiment conducted to evaluate the effects of applying the proposed expediting mechanism to the protein structural analysis problem addressed in [13]. The study presented in [13] is aimed at predicting the active sites of a new protein through aligning the tertiary structure of the new protein to that of the reference proteins, of which the active sites have been identified by biochemists. Experimental results reveal that a speedup of 4.8 to 10.3 times can be achieved with no significant impact on the prediction accuracy.

In the following part of this paper, section 2 elaborates the efficient kernel density estimation algorithm that the

\footnotetext{
${ }^{*}$ Corresponding author
} 
proposed expediting mechanism is based on. Section 3 reports the experiment conducted to evaluate the effects of exploiting the proposed expediting mechanism in a real protein structural analysis problem. Finally, concluding remarks are presented in section 4 .

\section{The efficient kernel density estimation algo- rithm}

The efficient kernel density estimation algorithm that forms the basis of the expediting mechanism proposed in this paper treats a given set of instances $\left\{\boldsymbol{s}_{1}, \boldsymbol{s}_{2}, \ldots, \boldsymbol{s}_{n}\right\}$ in the vector space as $n$ samples randomly taken from an unknown probability distribution and constructs an approximator of the following form for the unknown probability density function $f$ :

$$
\hat{f}(\boldsymbol{v})=\sum_{i=1}^{n} w_{i} \exp \left(-\frac{\left\|\boldsymbol{v}-\boldsymbol{s}_{\boldsymbol{i}}\right\|^{2}}{2 \sigma_{i}^{2}}\right) \cong f(\boldsymbol{v}),
$$

where $\boldsymbol{v}$ is a vector in the vector space and $\left\|\boldsymbol{v}-\boldsymbol{s}_{\boldsymbol{i}}\right\|$ is the distance between vectors $v$ and $s_{i}$. Accordingly, the task that the efficient kernel density estimation algorithm carries out is to determine the optimal values of $w_{i}$ and $\sigma_{i}$ in equation (1). In fact, the kernel density estimation problem described here can be transformed to a kernel smoothing problem [12], if we employ the following equation to estimate the values of $f$ at $\boldsymbol{s}_{i}, i=1,2, \ldots, n$ :

$$
f\left(s_{i}\right) \cong \frac{(k+1)}{n} \cdot\left[\frac{R\left(s_{i}\right)^{m} \pi^{\frac{m}{2}}}{\Gamma\left(\frac{m}{2}+1\right)}\right]^{-1}, \text { where }
$$

(i) $m$ is the dimension of the vector space;

(ii) $R\left(\boldsymbol{s}_{\boldsymbol{i}}\right)$ is the distance between instance $\boldsymbol{s}_{\boldsymbol{i}}$ and its $k$-th nearest neighbor;

(iii) $\frac{R\left(s_{\boldsymbol{i}}\right)^{m} \pi^{\frac{m}{2}}}{\Gamma\left(\frac{m}{2}+1\right)}$ is the volume of a hypersphere with radius $R\left(\boldsymbol{s}_{\boldsymbol{i}}\right)$ in an $m$-dimensional vector space;

(iv) $\Gamma(\cdot)$ is the Gamma function [2];

(v) $k$ is a parameter to be set by the user.

The efficient kernel density estimation algorithm employed in this paper is derived with some ideal assumptions. One may wonder whether these ideal assumptions may affect the accuracy of the algorithm. In fact, it has been shown in [10] that the kernel smoothing algorithm on which the efficient kernel density estimation algorithm employed in this paper is based is able to deliver the same level of accuracy as the other existing kernel smoothing algorithms. Another issue that needs to be addressed here concerns the use of $R\left(\boldsymbol{s}_{\boldsymbol{i}}\right)$ in equation (2). As $R\left(\boldsymbol{s}_{\boldsymbol{i}}\right)$ is determined by the distance of one single pair of instances, $s_{i}$ and its $k$-th nearest neighbor, the value tends to be unreliable, if the data set is noisy. In our implementation, we use $\bar{R}\left(\boldsymbol{s}_{\boldsymbol{i}}\right)$ defined in the following to replace $R\left(s_{i}\right)$ in equation (2),

$$
\bar{R}\left(\boldsymbol{s}_{\boldsymbol{i}}\right)=\frac{m+1}{m}\left(\frac{1}{k} \sum_{h=1}^{k}\left\|\hat{\boldsymbol{s}}_{\boldsymbol{h}}-\boldsymbol{s}_{\boldsymbol{i}}\right\|\right)
$$

, where $\hat{\boldsymbol{s}}_{\mathbf{1}}, \hat{\boldsymbol{s}}_{\mathbf{2}}, \ldots, \hat{\boldsymbol{s}}_{\boldsymbol{k}}$ are the $k$ nearest neighbors of $\boldsymbol{s}_{\boldsymbol{i}}$ and $m$ is the dimension of the vector space. The basis of employing $\bar{R}\left(\boldsymbol{s}_{\boldsymbol{i}}\right)$ is elaborated in Appendix A. Accordingly, we use the following equation in this paper to estimate the function values of $f$ at given instances:

$$
f\left(\boldsymbol{s}_{\boldsymbol{i}}\right) \cong \frac{(k+1)}{n} \cdot\left[\frac{\bar{R}\left(\boldsymbol{s}_{\boldsymbol{i}}\right)^{m} \pi^{\frac{m}{2}}}{\Gamma\left(\frac{m}{2}+1\right)}\right]^{-1} .
$$

Let us assume that we now want to derive an approximate probability density function of form shown in equation (1) for a given set of instances in an $m$-dimensional vector space. As shown in equation (1), the efficient kernel density estimation algorithm places one spherical Gaussian function at each instance. The challenge now is to figure out the optimal $w_{i}$ and $\sigma_{i}$ values for each Gaussian function in equation (1). For an instance $\boldsymbol{s}_{\boldsymbol{i}}$, the efficient kernel density estimation algorithm first conducts a mathematical analysis on a synthesized data set. The synthesized data set is derived from two ideal assumptions and serves as an analogy of the distribution of the instances in the proximity of $\boldsymbol{s}_{\boldsymbol{i}}$. The first assumption is that the sampling density in the proximity of $\boldsymbol{s}_{i}$ is sufficiently high and, as a result, the variation of the probability density function in the proximity of $\boldsymbol{s}_{\boldsymbol{i}}$ approaches 0 . The second assumption is that $\boldsymbol{s}_{\boldsymbol{i}}$ and its neighboring instances are evenly spaced by a distance determined by the value of the probability density function at $\boldsymbol{s}_{\boldsymbol{i}}$. Fig. 1 shows an example of the synthesized data set for a training sample in a 2-dimensional vector space. The details of the model are elaborated in the following.

(i) Instance $\boldsymbol{s}_{\boldsymbol{i}}$ is located at the origin and the neighboring instances are located at $\left(h_{1} \delta_{i}, h_{2} \delta_{i}, \ldots, h_{m} \delta_{i}\right)$, where $h_{1}$, $h_{2}, \ldots, h_{m}$ are integers and $\delta_{i}$ is the average distance between two adjacent instances in the proximity of $\boldsymbol{s}_{\boldsymbol{i}}$. How $\delta_{i}$ is determined will be elaborated later on.

(ii) The values of the probability density function at the instances in the synthesized data set, including $\boldsymbol{s}_{i}$, are all equal to $f\left(s_{i}\right)$. The value of $f\left(s_{i}\right)$ is estimated based on equation (3).

The efficient kernel density estimation algorithm begins with an analysis on the synthesized data set to figure out the values of $w_{i}$ and $\sigma_{i}$ that make function $g_{i}(\cdot)$ defined in the following virtually a constant function equal to $f\left(s_{i}\right)$,

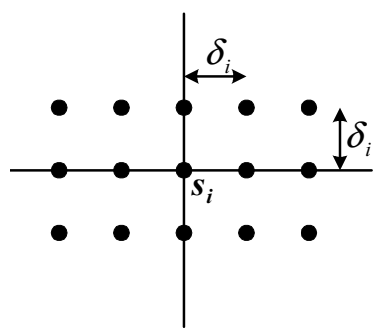

Fig. 1. An example of the synthesized data set for an instance in a 2-dimensional vector space. 


$$
\begin{aligned}
g_{i}(\boldsymbol{x}) & =w_{i}\left[\sum_{h_{1}=-\infty}^{\infty} \sum_{h_{2}=-\infty}^{\infty} \ldots \sum_{h_{m}=-\infty}^{\infty} \exp \left(-\frac{\left\|\boldsymbol{x}-\left(h_{1} \delta_{i}, h_{2} \delta_{i}, \ldots, h_{m} \delta_{i}\right)\right\|^{2}}{2 \sigma_{i}^{2}}\right)\right] \\
& \cong f\left(\boldsymbol{s}_{i}\right) .
\end{aligned}
$$

The motivation for conducting the analysis is to make $g_{i}(\boldsymbol{x})$ is a good approximator of $f(\boldsymbol{x})$ in the proximity of $\boldsymbol{s}_{\boldsymbol{i}}$ and its $k$ nearest neighboring instances.

Let

$$
q(y)=\sum_{h=-\infty}^{\infty} \exp \left(-\frac{\left(y-h \delta_{i}\right)^{2}}{2 \sigma_{i}^{2}}\right) .
$$

Then, we have

$$
\begin{aligned}
& \sum_{h_{1}=-\infty}^{\infty} \sum_{h_{2}=-\infty}^{\infty} \cdots \sum_{h_{m}=-\infty}^{\infty} \exp \left(-\frac{\left\|\boldsymbol{x}-\left(h_{1} \delta_{i}, h_{2} \delta_{i}, \ldots, h_{m} \delta_{i}\right)\right\|^{2}}{2 \sigma_{i}^{2}}\right) \\
= & \sum_{h_{1}=-\infty}^{\infty} \exp \left(-\frac{\left(x_{1}-h_{1} \delta_{i}\right)^{2}}{2 \sigma_{i}^{2}}\right) \cdot \sum_{h_{2}=-\infty}^{\infty} \exp \left(-\frac{\left(x_{2}-h_{2} \delta_{i}\right)^{2}}{2 \sigma_{i}^{2}}\right) \cdots \sum_{h_{m}=-\infty}^{\infty} \exp \left(-\frac{\left(x_{m}-h_{m} \delta_{i}\right)^{2}}{2 \sigma_{i}^{2}}\right)
\end{aligned}
$$

, where $\boldsymbol{x}=\left(x_{1}, x_{2}, \ldots, x_{m}\right)$. Therefore,

$$
\{\text { Minimum }[q(y)]\}^{m}
$$

$\leq \sum_{h_{1}=-\infty}^{\infty} \sum_{h_{2}=-\infty}^{\infty} \ldots \sum_{h_{m}=-\infty}^{\infty} \exp \left(-\frac{\left\|\boldsymbol{x}-\left(h_{1} \delta_{i}, h_{2} \delta_{i}, \ldots, h_{m} \delta_{i}\right)\right\|^{2}}{2 \sigma_{i}^{2}}\right)$

$\leq\{\operatorname{Maximum}[q(y)]\}^{m}$.

It is shown in Appendix B that, if $\sigma_{i}=\delta_{i}$, then we have

$$
\begin{aligned}
q(y) & \leq \lim _{n \rightarrow \infty} \underset{0 \leq j \leq n-1}{\operatorname{Maximum}}\left\{\sum_{h=-\infty}^{\infty} \exp \left(-\frac{1}{2}\left(\frac{j}{2 n}-h\right)^{2}\right), \sum_{h=-\infty}^{\infty} \exp \left(-\frac{1}{2}\left(\frac{j}{2 n}-h\right)^{2}\right)+\frac{\delta_{i}}{2 n} \theta\right\} \\
\cong 2.506628288 &
\end{aligned}
$$

where

$$
\begin{aligned}
\theta & =\left(\frac{-1}{\sigma_{i}}\right)\left(\frac{j}{2 n}\right) \exp \left[-\frac{1}{2}\left(\frac{j}{2 n}\right)^{2}\right] \\
& +\left(\frac{-1}{\sigma_{i}}\right)\left(\frac{j}{2 n}-1\right) \exp \left[-\frac{1}{2}\left(\frac{j}{2 n}-1\right)^{2}\right] \\
& +\left(\frac{-1}{\sigma_{i}}\right) \sum_{\substack{h=-\infty \\
h \neq 0,1}}^{\infty}\left(\frac{(j+1)}{2 n}-h\right) \exp \left[-\frac{1}{2}\left(\frac{(j+1)}{2 n}-h\right)^{2}\right] .
\end{aligned}
$$

Furthermore, we have

$$
\begin{aligned}
q(y) & \geq \lim _{n \rightarrow \infty} \operatorname{Minimum}_{0 \leq j \leq n-1}\left\{\sum_{h=-\infty}^{\infty} \exp \left(-\frac{1}{2}\left(\frac{j}{2 n}-h\right)^{2}\right), \sum_{h=-\infty}^{\infty} \exp \left(-\frac{1}{2}\left(\frac{j}{2 n}-h\right)^{2}\right)+\frac{\delta_{i}}{2 n} \rho\right\} \\
& \cong 2.506628261,
\end{aligned}
$$

where

$$
\begin{aligned}
\rho & =\left(\frac{-1}{\sigma_{i}}\right)\left(\frac{j+1}{2 n}\right) \exp \left[-\frac{1}{2}\left(\frac{j+1}{2 n}\right)^{2}\right] \\
& +\left(\frac{-1}{\sigma_{i}}\right)\left(\frac{j+1}{2 n}-1\right) \exp \left[-\frac{1}{2}\left(\frac{j+1}{2 n}-1\right)^{2}\right] \\
& +\left(\frac{-1}{\sigma_{i}}\right) \sum_{\substack{h=-\infty \\
h \neq 0,1}}^{\infty}\left(\frac{j}{2 n}-h\right) \exp \left[-\frac{1}{2}\left(\frac{j}{2 n}-h\right)^{2}\right] .
\end{aligned}
$$

Therefore, with $\sigma_{i}=\delta_{i}, q(y)$ is bound by $2.5066282745 \pm$ $1.34 \times 10^{-8}$ and $g_{i}(\boldsymbol{x})$ defined in equation (4) is virtually a constant function. In fact, we can apply basically the same procedure presented in Appendix B to find the upper bounds and lower bounds of $q(y)$ with alternative $\frac{\sigma_{i}}{\delta_{i}}$ ratios. As Table 1 reveals, the variation of $q(y)$ becomes smaller, if $\beta=\frac{\sigma_{i}}{\delta_{i}}$ is set to a larger value. However, the magnitude of the variation of $q(y)$ is not the only concern. If $\beta$ is set to a large value, then a smoothing effect is induced. According to our previous study $[10,11]$, setting $\beta$ to a value around 1 is in general a good choice with respect to approximation accuracy.

Table 1. The bounds of function $q(y)$ defined in equation (4) with alternative $\frac{\sigma_{i}}{\delta_{i}}$ ratios.

\begin{tabular}{|c|c|}
\hline$\beta=\frac{\sigma_{i}}{\delta_{i}}$ & Bounds of $q(y)$ \\
\hline 0.5 & $1.253314144 \pm 1.80 \times 10^{-2}$ \\
\hline 1.0 & $2.506628275 \pm 1.34 \times 10^{-8}$ \\
\hline 1.5 & $3.759942412 \pm 2.94 \times 10^{-11}$ \\
\hline
\end{tabular}

As it has been shown that, with an appropriate $\frac{\sigma_{i}}{\delta_{i}}$ ratio, $g_{i}(\boldsymbol{x})$ defined in equation (4) is virtually a constant function, the next thing to do is to figure out the appropriate value of $w_{i}$ that makes $g_{i}(\boldsymbol{x})$ approximately equal to $f\left(\boldsymbol{s}_{\boldsymbol{i}}\right)$. We have $g_{i}\left(\boldsymbol{s}_{\boldsymbol{i}}\right)=g_{i}(0, \ldots, 0)$

$$
\begin{aligned}
& =w_{i}\left(\sum_{h_{1}=-\infty}^{\infty} \sum_{h_{2}=-\infty}^{\infty} \cdots \sum_{h_{m}=-\infty}^{\infty} \exp \left(-\frac{\left(h_{1}^{2}+h_{2}^{2}+\cdots+h_{m}^{2}\right) \delta_{i}^{2}}{2 \sigma_{i}^{2}}\right)\right) \\
& =w_{i}\left(\sum_{h=-\infty}^{\infty} \exp \left(-\frac{h^{2}}{2 \beta^{2}}\right)\right)^{m},
\end{aligned}
$$

where $\beta=\frac{\sigma_{i}}{\delta_{i}}$. Therefore, we need to set $w_{i}$ as follows, in order to make $g_{i}(\boldsymbol{x})$ a good approximator of $f(\boldsymbol{x})$ in the proximity of $\boldsymbol{s}_{\boldsymbol{i}}$ :

$$
w_{i}\left(\sum_{h=-\infty}^{\infty} \exp \left(-\frac{h^{2}}{2 \beta^{2}}\right)\right)^{m}=f\left(\boldsymbol{s}_{\boldsymbol{i}}\right) .
$$

If we employ equation (3) to estimate the value of $f\left(\boldsymbol{s}_{i}\right)$, then we have

$$
w_{i}=\frac{\left(k_{1}+1\right) \cdot \Gamma\left(\frac{m}{2}+1\right)}{\lambda^{m} \cdot\left|S_{j}\right| \cdot \bar{R}\left(s_{i}\right)^{m} \cdot \pi^{\frac{m}{2}}}, \text { where } \lambda=\sum_{h=-\infty}^{\infty} \exp \left(-\frac{h^{2}}{2 \beta^{2}}\right) .
$$

So far, we have figured out that if we an appropriate ratio of $\beta=\frac{\sigma_{i}}{\delta_{i}}$ and set $w_{i}$ according to equation (8), we can make $g_{i}(\boldsymbol{x})$ a good approximator of $f(\boldsymbol{x})$ in the proximity of $\boldsymbol{s}_{\boldsymbol{i}}$. The only remaining issue is to derive a closed form of $\sigma_{i}$. In this paper, $\delta_{i}$ is set to the average distance between two 
adjacent class- $j$ training samples in the proximity of sample $\boldsymbol{s}_{\boldsymbol{i}}$. In an $m$-dimensional vector space, the number of uniformly distributed samples, $N$, in a hypercube with volume $V$ can be computed by $N \cong \frac{V}{\alpha^{m}}$, where $\alpha$ is the spacing between two adjacent samples. Accordingly, we set

$$
\delta_{i}=\frac{\bar{R}\left(\boldsymbol{s}_{\boldsymbol{i}}\right) \sqrt{\pi}}{\sqrt[m]{(k+1) \Gamma\left(\frac{m}{2}+1\right)}} .
$$

Finally, with equations (8) and (9), we have the following approximate probability density function for the given set of instances:

$$
\begin{aligned}
\hat{f}(\boldsymbol{v}) & =\sum_{i=1}^{n} w_{i} \exp \left(-\frac{\left\|\boldsymbol{v}-\boldsymbol{s}_{\boldsymbol{i}}\right\|^{2}}{2 \sigma_{i}^{2}}\right) \\
& =\sum_{i=1}^{n} \frac{(k+1) \cdot \Gamma\left(\frac{m}{2}+1\right)}{\lambda^{m} \cdot\left|S_{j}\right| \cdot \bar{R}\left(\boldsymbol{s}_{\boldsymbol{i}}\right)^{m} \cdot \pi^{\frac{m}{2}}} \exp \left(-\frac{\left\|\boldsymbol{v}-\boldsymbol{s}_{\boldsymbol{i}}\right\|^{2}}{2 \sigma_{i}^{2}}\right) \\
& =\frac{1}{n} \sum_{i=1}^{n}\left(\frac{\beta}{\lambda \cdot \sigma_{i}}\right)^{m} \exp \left(-\frac{\left\|\boldsymbol{v}-\boldsymbol{s}_{\boldsymbol{i}}\right\|^{2}}{2 \sigma_{i}^{2}}\right)
\end{aligned}
$$

where

(i) $v$ is a vector in the $m$-dimensional vector space,

$$
\begin{aligned}
& \text { (ii) } \sigma_{i}=\beta \delta_{i}=\beta \frac{\bar{R}\left(s_{i}\right) \sqrt{\pi}}{\sqrt[m]{(k+1) \Gamma\left(\frac{m}{2}+1\right)}}, \\
& \text { (iii) } \lambda=\sum_{h=-\infty}^{\infty} \exp \left(-\frac{h^{2}}{2 \beta^{2}}\right) .
\end{aligned}
$$

One interesting observation is that, regardless of which $\beta=\frac{\sigma_{i}}{\delta_{i}}$ ratio is employed, we have $\frac{\lambda}{\beta} \cong \sqrt{2 \pi}$. If this observation can be proved to be generally correct, then we can further simplify equation (10) and obtain

$$
\hat{f}(\boldsymbol{v})=\frac{1}{n} \sum_{i=1}^{n}\left(\frac{1}{\sqrt{2 \pi} \cdot \sigma_{i}}\right)^{m} \exp \left(-\frac{\left\|\boldsymbol{v}-\boldsymbol{s}_{\boldsymbol{i}}\right\|^{2}}{2 \sigma_{i}^{2}}\right) .
$$

As far as the time complexity of the proposed kernel density estimation algorithm is concerned, let us first address a useful observation. The observation is that the Gaussian functions in equations (10) and (11) decrease exponentially as the value of the distance term increases. Therefore, when employing either equation (10) or (11) to compute an approximate function value at a point in the vector space, we can essentially ignore the Gaussian functions far away from the point. Accordingly, in our implementation, we simply include only a fixed number of nearest Gaussian functions, when invoking equations (10) and (11). In following discussion, the number of nearest Guassian functions included in evaluating equations (10) and (11) is denoted by $k^{\prime}$. With this practice employed and with the kd-tree structure employed to search the $k$ nearest neighbors of an instance, it can be shown that the average time complexity of the kernel density estimation algorithm is
$\mathrm{O}(n \log n)$, where the dimension of the vector space, i.e. $m$ in equations (10) and (11), is considered as a constant. The detailed analysis of the time complexity can be found in [10, $11]$.

\section{Application and experimental results}

In this section, we will evaluate the effects of applying the expediting mechanism proposed in this paper to the real protein structural analysis problem addressed in [13]. The study presented in [13] is aimed at predicting the active sites of a new protein through aligning the tertiary structure of the new protein to that of the reference proteins, of which the active sites have been identified by biochemists. With the prediction results, the biochemists then can narrow down their attention to the predicted regions and conduct comprehensive studies for final verification. The prediction mechanism is based on the generally accepted hypothesis in the bioinformatics research community that proteins with similar tertiary structures must have similar biological functions [9].

The prediction mechanism proposed in [13] invokes the geometric hashing algorithm in computer graphics to carry out structural alignment and exploits the fact that the two backbone bonds connected to the alpha carbon of an amino acid residue span almost at the same degree regardless of the type of the amino acid [7]. Accordingly, the search domain of the geometric hashing algorithm is narrowed down to only the coordinate systems defined by the two backbone bonds connected to the alpha carbon of a residue. As a result, the time complexity of the prediction mechanism is $\mathrm{O}\left(n_{1} n_{2}\left(n_{1}+q\right)\right)$, where $n_{1}$ and $n_{2}$ are the number of residues in the protein to be studied and in the reference protein, respectively, and $q$ is the number of residues in the active sites of the reference protein.

In the following discussion, the efficient kernel density estimation algorithm presented in section 2 is invoked to expedite the prediction mechanism proposed in [13]. The expediting mechanism exploits the observation that the active sites of a protein are in general located in the caves of the protein tertiary structure. Accordingly, the search domain of the geometric hashing algorithm is further narrowed down to only the coordinate systems defined by the residues in the caves of the proteins. Fig. 2 depicts a 2dimensional example to illustrate the effect of the expediting mechanism. In this example, a 2-dimensional object is composed of a number of primitive instances represented by dots in the figure. The kernel density estimation based expediting mechanism proposed in this paper treats the instances in the vector space as $n$ random samples taken from an unknown probability distribution. If the probability density function corresponding to the distribution is a smooth and continuous function in the vector space, then we can expect that the function values on the contour of the object will generally be smaller than the function values in the center of the object. Accordingly, this paper proposes to employ equation (10) to obtain an approximate probability 
density function and then set a threshold to distinguish those instances on the contour of the object and those in the center. Fig. 2(a) shows the effect achieved with the kernel density estimation based mechanism.

With the instances on the contour of the object been successfully identified as shown in Fig. 2(a), the next task is to further classify these instances depending on whether the instance is located in a cave of the object or not. This task can be carried out by applying equation (10) again but with a larger $\beta$ value. Applying equation (10) with a larger $\beta$ value implies that the approximate probability density function obtained is smoother. As a result, the function values at those instances that are located in a cave will be generally higher than the function values at those instances that are on the contour of the protein but not in a cave. Accordingly, a threshold can be set to classify these instances. Fig. 2(b) shows the final result obtained in this example and Fig. 3 shows the pseudo-code of the kernel density estimation based filtering mechanism.

In the following experiment, the filtering mechanism presented in Fig. 3 is invoked to identify the atoms located in a cave of a protein tertiary structure. In this filtering process, only the nitrogen and carbon atoms on the backbone of the residues are included and a residue is said to be in a cave if two out of its three backbone atoms are in a cave. With the output of the filtering process, the geometric hashing algorithm that is invoked to carry out structural alignment then narrows down its search domain by examining only the coordinate systems defined by the residues located in a cave. Table 2 shows how the parameters are set in the filtering process and the criteria for successful alignment of two atoms in the geometric hashing algorithm.

Instance at which the value of the approximate probability density function does exceed the threshold in the first run.

O Instance at which the value of the approximate probability density function does not exceed the threshold in the first run.

O Instance identified as in a cave.
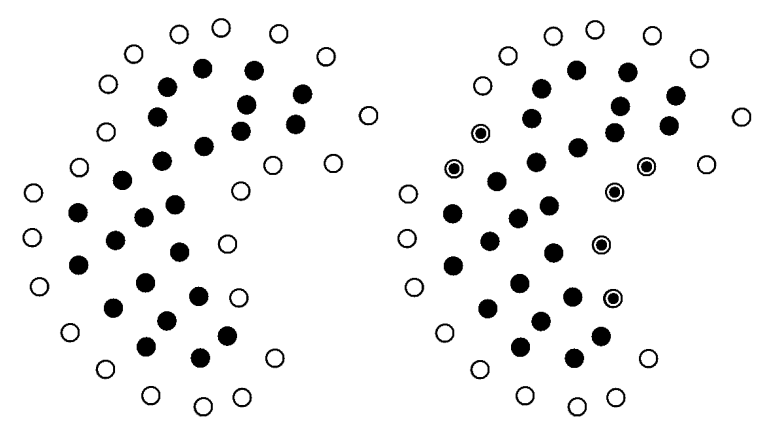

(a) The effect after first run. (b) The effect after the second run.

Fig. 2. An example that illustrates the effect of the proposed kernel density estimation based filtering mechanism.
Algorithm kernel density estimation based filtering

Input: A set $S=\left\{s_{1}, s_{2}, \ldots, s_{n}\right\}$ of instances in the vector space and parameters $\beta_{1}, \beta_{2}, k, k^{\prime}, r_{1}$, and $r_{2}$.

Output: $\hat{S}$, a subset of $S$.

Set $\hat{S} \leftarrow S$.

For each $s_{i} \in S$ do the following:

Compute $\hat{f}\left(s_{i}\right)$ according to equation (10) with $\beta=\beta_{1}$.

Set $w \leftarrow \max _{1 \leq i \leq n}\left\{\hat{f}\left(s_{i}\right)\right\}$.

For each $s_{i} \in S$ do the following:

$$
\text { If } \hat{f}\left(s_{i}\right) \leq r_{1} \cdot \frac{1}{2} \cdot w \text {, then } \hat{S} \leftarrow \hat{S}-\left\{s_{i}\right\} \text {. }
$$

For each $s_{i} \in S$ do the following:

Compute $\hat{f}\left(s_{i}\right)$ according to equation (10) with $\beta=\beta_{2}$.

Set $w \leftarrow \max _{1 \leq i \leq n}\left\{\hat{f}\left(s_{i}\right)\right\}$.

For each $s_{i} \in S$ do the following:

$$
\text { If } \hat{f}\left(s_{i}\right) \leq r_{2} \cdot \frac{1}{2} \cdot w \text {, then } \hat{S} \leftarrow \hat{S}-\left\{s_{i}\right\} \text {. }
$$

$\operatorname{Return}(\hat{S})$.

Fig. 3. The pseudo-code of the kernel density estimation based filtering mechanism.

Table 2. Parameter settings in the experiment.

\begin{tabular}{|c|c|}
\hline Parameter & Value \\
\hline$\beta_{1}$ in pseudo code & 0.45 \\
\hline$\beta_{2}$ in pseudo code & 0.9 \\
\hline$k$ in equation $(2)$ & 30 \\
\hline$k^{\prime}$ for evaluating equation $(10)$ & 30 \\
\hline$r_{1}$ in pseudo code & 1.33 \\
\hline$r_{2}$ in pseudo code & 1.25 \\
\hline
\end{tabular}

(a) Parameter settings for the kernel density estimation based filtering mechanism.

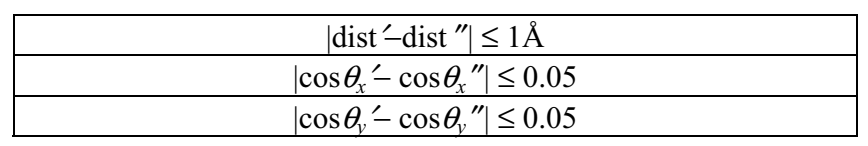

dist' and dist " are the distances from the origin to the two atoms, respectively.

$\theta_{x}{ }^{\prime}$ and $\theta_{x}{ }^{\prime \prime}$ are the angles between axis $x$ and the vectors to the two atoms, respectively.

$\theta_{y}{ }^{\prime}$ and $\theta_{y}{ }^{\prime \prime}$ are the angles between axis $y$ and the vectors to the two atoms, respectively.

(b) Criteria for successful alignment of two atoms in the geometric hashing algorithm.

In the experiment, three proteins, $1 \mathbf{h d z}, \mathbf{1 b 1 5}$, and $1 \mathrm{e} 7 \mathrm{~m}$, from the PDB database are used as the reference proteins. For each reference protein, we select additional 5 proteins from the same family as the test samples. Table 3(a) gives the number of residues that are identified as in the caves of each protein and Table 3(b) presents the effects of the kernel density estimation based filtering mechanism on prediction accuracy and execution time. In Table 3(b), a residue in the test protein is said to be successfully aligned with a 
Table 3. Experimental results.

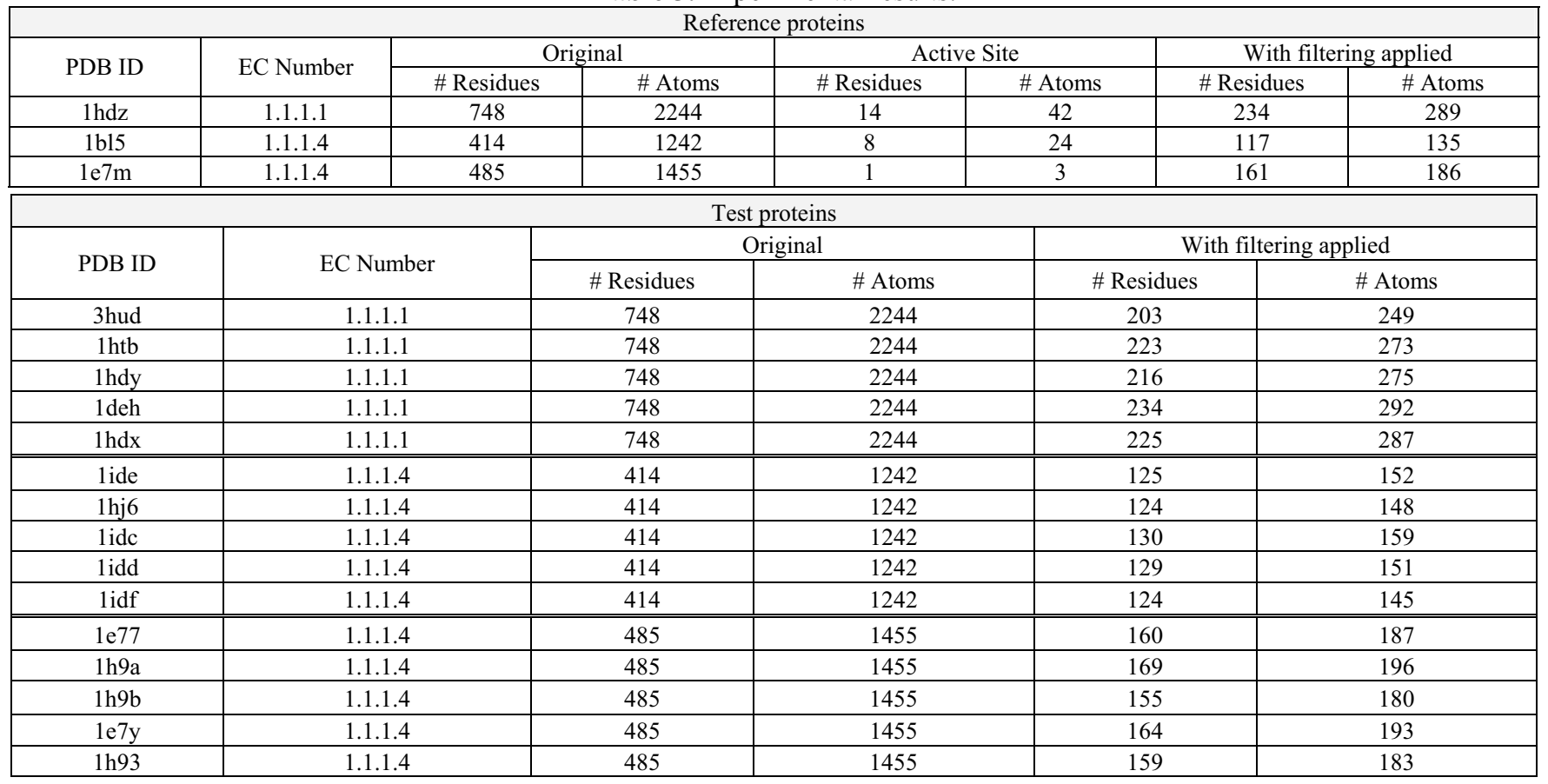

(a) The number of atoms and the number of residues that are identified as in the caves of each protein.

\begin{tabular}{|c|c|c|c|c|c|c|c|c|c|c|}
\hline \multirow[b]{2}{*}{$\begin{array}{c}\text { Ref } \\
\text { Protein }\end{array}$} & \multirow[b]{2}{*}{$\begin{array}{c}\text { Test } \\
\text { Protein }\end{array}$} & \multicolumn{2}{|c|}{ Active site } & \multicolumn{3}{|c|}{ Geometric hashing without filtering } & \multicolumn{4}{|c|}{ Geometric Hashing with filtering applied } \\
\hline & & \# Residues & \# Atoms & $\begin{array}{l}\text { Execution } \\
\text { time of } \\
\text { geometric } \\
\text { hashing in } \\
\text { seconds }\end{array}$ & $\begin{array}{l}\text { \# of residues in } \\
\text { the active sites } \\
\text { that are success- } \\
\text { fully aligned }\end{array}$ & $\begin{array}{l}\# \text { atoms in the } \\
\text { active sites } \\
\text { that are } \\
\text { successfully } \\
\text { aligned }\end{array}$ & $\begin{array}{c}\text { Execution } \\
\text { time of } \\
\text { filtering in } \\
\text { seconds }\end{array}$ & $\begin{array}{c}\text { Execution time } \\
\text { of geometric } \\
\text { hashing in } \\
\text { seconds }\end{array}$ & $\begin{array}{c}\text { \# of residues in the } \\
\text { active sites that } \\
\text { are } \\
\text { successfully } \\
\text { aligned. }\end{array}$ & $\begin{array}{l}\text { \# of atoms in the } \\
\text { active sites } \\
\text { that are } \\
\text { successfully } \\
\text { aligned. }\end{array}$ \\
\hline \multirow{5}{*}{ 1hdz } & 3hud & 14 & 42 & 86.24 & 14 & \begin{tabular}{|l|}
41 \\
\end{tabular} & 0.79 & 7.57 & 14 & 41 \\
\hline & $1 \mathrm{htb}$ & 14 & 42 & 84.88 & 14 & 42 & 0.78 & 8.27 & 14 & 42 \\
\hline & 1hdy & 14 & 42 & 85.11 & 14 & 42 & 0.79 & 8.06 & 14 & 42 \\
\hline & $1 \mathrm{deh}$ & 14 & 42 & 85.42 & 14 & 42 & 0.83 & 8.73 & 14 & 42 \\
\hline & 1hdx & 14 & 42 & 84.98 & 14 & 41 & 0.83 & 8.38 & 14 & 40 \\
\hline \multirow{5}{*}{$1 \mathrm{bl} 5$} & lide & 8 & 24 & 12.82 & 8 & 24 & 0.29 & 1.22 & 8 & 24 \\
\hline & 1hj6 & 8 & 24 & 12.91 & 8 & 23 & 0.29 & 1.22 & 7 & 21 \\
\hline & lide & 8 & 24 & 12.82 & 8 & 24 & 0.3 & 1.27 & 8 & 24 \\
\hline & lidd & 8 & 24 & 12.85 & 8 & 24 & 0.29 & 1.26 & 8 & 23 \\
\hline & lidf & 8 & 24 & 12.77 & 8 & 24 & 0.29 & 1.22 & 8 & 24 \\
\hline \multirow{5}{*}{$1 \mathrm{e} 7 \mathrm{~m}$} & $1 \mathrm{e} 77$ & 1 & 3 & 5.61 & 1 & 3 & 0.37 & 0.80 & 1 & 3 \\
\hline & $1 \mathrm{~h} 9 \mathrm{a}$ & 1 & 3 & 5.66 & 1 & 3 & 0.35 & 0.84 & 1 & 3 \\
\hline & $1 \mathrm{~h} 9 \mathrm{~b}$ & 1 & 3 & 5.68 & 1 & 3 & 0.36 & 0.78 & 1 & 3 \\
\hline & $1 \mathrm{e} 7 \mathrm{y}$ & 1 & 3 & 5.65 & 1 & 3 & 0.36 & 0.81 & 1 & 3 \\
\hline & $1 \mathrm{~h} 93$ & 1 & 3 & 5.59 & 1 & 3 & 0.37 & 0.80 & 1 & 3 \\
\hline
\end{tabular}

(b) The effects of the kernel density estimation based filtering mechanism on prediction accuracy and execution time.

residue in the reference protein, if two out of the nitrogen and two carbon atoms on the backbone of the residue are successfully aligned with the corresponding atoms in the reference protein. As the experimental results reveal, a speedup of 4.8 to 10.3 times can be achieved with the kernel density estimation based filtering mechanism and no significant impact on the prediction accuracy is observed.

\section{Conclusion}

In this paper, a kernel density estimation based mechanism aimed at expediting protein structural analysis is proposed. This study has been motivated by the observation that many protein structural analysis algorithms suffer high time complexity and only the residues and atoms on the contour of a protein are essential for determining the functions of the protein and how it could interact with the other proteins. The expediting mechanism proposed in this paper invokes a novel kernel density estimation algorithm to filter the atoms and residues in the proteins to be analyzed. The average time complexity of the filtering mechanism is $\mathrm{O}(n \log n)$. This paper reports an experiment conducted to evaluate the effects of applying the proposed expediting mechanism to a real protein structural analysis problem. The real problem concerns how to predict the active sites of a new protein through aligning the tertiary structure of the new protein to that of the reference proteins. Experimental 
results reveal that a speedup of 4.8 to 10.3 times can be achieved with the proposed expediting mechanism and no significant impact on the prediction accuracy is observed.

As the kernel density estimation based filtering algorithm performs quite effectively in the protein structural analysis problem addressed in this paper, it is of interest to investigate how this algorithm and its alternative versions can be applied to other protein structural analysis problems. There are several possibilities that deserve continuous investigation, including general protein structural alignment, protein structures classification, and folds prediction.

\section{Appendix A}

Assume that $\hat{\boldsymbol{s}}_{1}, \hat{\boldsymbol{s}}_{2}, \ldots, \hat{\boldsymbol{s}}_{k}$ are the $k$ nearest neighbors of $\boldsymbol{s}_{\boldsymbol{i}}$. If $k$ is sufficiently large and the distribution of these $k$ instances in the vector space is uniform then we have

$$
k \approx \frac{\rho R\left(s_{i}\right)^{m} \pi^{\frac{m}{2}}}{\Gamma\left(\frac{m}{2}+1\right)},
$$

where $\rho$ is the local density of instances $\hat{\boldsymbol{s}}_{1}, \hat{\boldsymbol{s}}_{2}, \ldots, \hat{\boldsymbol{s}}_{k}$ in the proximity of $s_{i}$. Furthermore, we have

$$
\begin{aligned}
& \sum_{h=1}^{k}\left\|\hat{\boldsymbol{s}}_{\boldsymbol{h}}-\boldsymbol{s}_{\boldsymbol{i}}\right\| \approx \int_{0}^{R\left(s_{i}\right)} \rho\left(\frac{2 r^{m-1} \pi^{\frac{m}{2}}}{\Gamma\left(\frac{m}{2}\right)}\right) r d r \\
= & \frac{2 \rho R\left(\boldsymbol{s}_{\boldsymbol{i}}\right)^{m+1} \pi^{\frac{m}{2}}}{(m+1) \Gamma\left(\frac{m}{2}\right)},
\end{aligned}
$$

where $\frac{2 r^{m-1} \pi^{\frac{m}{2}}}{\Gamma\left(\frac{m}{2}\right)}$ is the surface area of a hypersphere with radius $r$ in an $m$-dimensional vector space. Therefore, we have

$$
R\left(\boldsymbol{s}_{\boldsymbol{i}}\right)=\frac{m+1}{m} \cdot \frac{1}{k} \sum_{h=1}^{k_{1}}\left\|\hat{\boldsymbol{s}}_{\boldsymbol{h}}-\boldsymbol{s}_{\boldsymbol{i}}\right\| .
$$

The right-hand side of the equation above is then employed in this paper to estimate $R\left(\boldsymbol{s}_{i}\right)$.

\section{Appendix B}

Let $q(y)=\sum_{h=-\infty}^{\infty} \exp \left(-\frac{(y-h \delta)^{2}}{2 \sigma^{2}}\right)$, where $\delta \in \boldsymbol{R}$ and $\sigma \in$

$\boldsymbol{R}$ are two coefficients and $y \in \boldsymbol{R}$. We have

$$
q^{\prime}(y)=\frac{d q(y)}{d y}=\left(-\frac{1}{\sigma_{i}^{2}}\right) \sum_{h=-\infty}^{\infty}(y-h \delta) \exp \left(-\frac{(y-h \delta)^{2}}{2 \sigma^{2}}\right) .
$$

Since $q(y)$ is a symmetric and periodical function, if we want to find the global maximum and minimum values of $q(y)$, we only need to analyze $q(y)$ within interval $\left[0, \frac{\delta}{2}\right]$.

Let $y_{0} \in\left[0, \frac{\delta}{2}\right)$ and $y_{0}=\frac{\delta}{2} \cdot \frac{j}{n}+\varepsilon$, where $n \geq 1$ and $0 \leq j$ $<n-1$ are integers, and $0 \leq \varepsilon<\frac{\delta}{2 n}$. We have

$$
q\left(y_{0}\right)=q\left(\frac{j \delta}{2 n}\right)+\int_{\frac{j \delta}{2 n}}^{\frac{j \delta}{2 n}+\varepsilon} q^{\prime}(t) d t .
$$

Let us consider the special case with $\sigma=\delta$. Then, we have

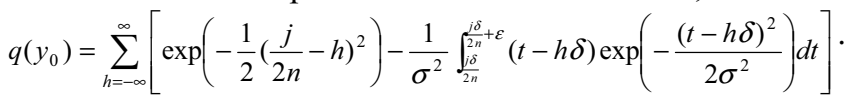

Let $\quad r(h)=-\frac{1}{\sigma^{2}} \int_{\frac{j \delta}{2 n}}^{\frac{j \delta}{2 n}+\varepsilon}(t-h \delta) \exp \left(-\frac{(t-h \delta)^{2}}{2 \sigma^{2}}\right) d t$

Since $\frac{-1}{\sigma^{2}}(t-h \delta) \exp \left(-\frac{(t-h \delta)^{2}}{2 \sigma^{2}}\right)$ is an increasing function for $t \in[(h-1) \delta,(h+1) \delta]$ and is a decreasing function for $t \notin[(h-1) \delta,(h+1) \delta]$, we have

$$
\text { (i) } \begin{aligned}
r(0) & \leq \varepsilon\left(\frac{-1}{\sigma^{2}}\right)\left(\frac{j \delta}{2 n}\right) \exp \left[-\frac{1}{2 \sigma^{2}}\left(\frac{j \delta}{2 n}\right)^{2}\right] \\
& =\varepsilon\left(\frac{-1}{\sigma}\right)\left(\frac{j}{2 n}\right) \exp \left[-\frac{1}{2}\left(\frac{j}{2 n}\right)^{2}\right] ; \\
\text { (ii) } r(1) & \leq \varepsilon\left(\frac{-1}{\sigma^{2}}\right)\left(\frac{j \delta}{2 n}-\delta\right) \exp \left[-\frac{1}{2 \sigma^{2}}\left(\frac{j \delta}{2 n}-\delta\right)^{2}\right] \\
& =\varepsilon\left(\frac{-1}{\sigma}\right)\left(\frac{j}{2 n}-1\right) \exp \left[-\frac{1}{2}\left(\frac{j}{2 n}-1\right)^{2}\right] ;
\end{aligned}
$$

(iii) for $h \neq 0$ and $h \neq 1$,

$$
\begin{aligned}
r(h) & \leq \varepsilon\left(\frac{-1}{\sigma^{2}}\right)\left(\frac{(j+1) \delta}{2 n}-h \delta\right) \exp \left[-\frac{1}{2 \sigma^{2}}\left(\frac{(j+1) \delta}{2 n}-h \delta\right)^{2}\right] \\
& =\varepsilon\left(\frac{-1}{\sigma}\right)\left(\frac{(j+1)}{2 n}-h\right) \exp \left[-\frac{1}{2}\left(\frac{(j+1)}{2 n}-h\right)^{2}\right] .
\end{aligned}
$$

Therefore,

$$
\begin{aligned}
q\left(y_{0}\right) & =\sum_{h=-\infty}^{\infty}\left[\exp \left(-\frac{1}{2}\left(\frac{j}{2 n}-h\right)^{2}\right)+r(h)\right] \\
& \leq\left[\sum_{h=-\infty}^{\infty} \exp \left(-\frac{1}{2}\left(\frac{j}{2 n}-h\right)^{2}\right)\right]+\varepsilon \theta, \text { where } \\
\theta & =\left(\frac{-1}{\sigma}\right)\left(\frac{j}{2 n}\right) \exp \left[-\frac{1}{2}\left(\frac{j}{2 n}\right)^{2}\right] \\
& +\left(\frac{-1}{\sigma}\right)\left(\frac{j}{2 n}-1\right) \exp \left[-\frac{1}{2}\left(\frac{j}{2 n}-1\right)^{2}\right] \\
& +\left(\frac{-1}{\sigma}\right) \sum_{\substack{h=-\infty \\
h \neq 0,1}}^{\infty}\left(\frac{(j+1)}{2 n}-h\right) \exp \left[-\frac{1}{2}\left(\frac{(j+1)}{2 n}-h\right)^{2}\right] .
\end{aligned}
$$

If $\theta \geq 0$, then we have for any $0 \leq \varepsilon<\frac{\delta}{2 n}$ 


$$
\begin{aligned}
& {\left[\sum_{h=-\infty}^{\infty} \exp \left(-\frac{1}{2}\left(\frac{j}{2 n}-h\right)^{2}\right)\right]+\varepsilon \theta } \\
\leq & {\left[\sum_{h=-\infty}^{\infty} \exp \left(-\frac{1}{2}\left(\frac{j}{2 n}-h\right)^{2}\right)\right]+\frac{\delta}{2 n} \theta . }
\end{aligned}
$$

On the other hand, if $\theta<0$, then we have for any

$$
\begin{aligned}
0 & \leq \varepsilon<\frac{\delta_{i}}{2 n} \\
& {\left[\sum_{h=-\infty}^{\infty} \exp \left(-\frac{1}{2}\left(\frac{j}{2 n}-h\right)^{2}\right)\right]+\varepsilon \theta } \\
& \leq\left[\sum_{h=-\infty}^{\infty} \exp \left(-\frac{1}{2}\left(\frac{j}{2 n}-h\right)^{2}\right)\right]
\end{aligned}
$$

Combining equations (A.1) and (A.2), we obtain, for all $y$ $\in\left[0, \frac{\delta}{2}\right]$,

$$
q(y) \leq \lim _{n \rightarrow \infty} \operatorname{Maximum}_{0 \leq \leqslant \leq k n-1}\left\{\sum_{h=-\infty}^{\infty} \exp \left(-\frac{1}{2}\left(\frac{j}{2 n}-h\right)^{2}\right), \sum_{h=-\infty}^{\infty} \exp \left(-\frac{1}{2}\left(\frac{j}{2 n}-h\right)^{2}\right)+\frac{\delta}{2 n} \theta\right\} .
$$

Similarly, we can show that

$$
q(y) \geq \lim _{n \rightarrow \infty} \operatorname{Minimum}_{0 \leq \leq \leq n-1}\left\{\sum_{h=-\infty}^{\infty} \exp \left(-\frac{1}{2}\left(\frac{j}{2 n}-h\right)^{2}\right), \sum_{h=-\infty}^{\infty} \exp \left(-\frac{1}{2}\left(\frac{j}{2 n}-h\right)^{2}\right)+\frac{\delta}{2 n} \rho\right\}
$$

, where

$$
\begin{aligned}
\rho & =\left(\frac{-1}{\sigma}\right)\left(\frac{j+1}{2 n}\right) \exp \left[-\frac{1}{2}\left(\frac{j+1}{2 n}\right)^{2}\right] \\
& +\left(\frac{-1}{\sigma}\right)\left(\frac{j+1}{2 n}-1\right) \exp \left[-\frac{1}{2}\left(\frac{j+1}{2 n}-1\right)^{2}\right] \\
& +\left(\frac{-1}{\sigma}\right) \sum_{\substack{h=-\infty \\
h \neq 0,1}}^{\infty}\left(\frac{j}{2 n}-h\right) \exp \left[-\frac{1}{2}\left(\frac{j}{2 n}-h\right)^{2}\right]
\end{aligned}
$$

If we set $n=100,000$, then we have

$$
2.506628261 \leq q(y) \leq 2.506628288 \text {, for } y \in\left[0, \frac{\delta}{2}\right] \text {. }
$$

\section{References:}

[1] Altschul S.F. (1991) Amino acid substitution matrices from an information heoretic perspective. J. Mol. Biol. 219, 555-565.

[2] Artin, E. (1964) The Gamma Function, New York, Holt, Rinehart, and Winston.
[3] Berman, H.M., Westbrook, J., Feng, Z., Gilliland, G., Bhat, T.N., Weissig, H., Shindyalov, I.N., Bourne, P.E. (2000) The Protein Data Bank. Nucleic Acids Research, 28, 235-242.

[4] Edelsbrunner, H. and Mucke, E. P. (1994) Threedimensional alpha shapes. ACM Trans. Graphics, 13, 43-72.

[5] Haim, J. W. (1997) Geometric hashing: an overview. IEEE Comput. Sc. and Eng. 4, 10-21.

[6] Kabsch, W. and Sander, C. (1983) Dictionary of Protein Secondary Structure: Pattern Recognition of Hydrogen-Bonded and Geometrical Features, Biopolymers, 22, 2577-2637.

[7] Krane, D. E.and Raymer, M. L. (2002) Fundamental Concepts of Bioinformatics, Benjamin Cummings.

[8] Lamdan, Y. and Wolfson, H. (1988) Geometric Hashing: A General and Efficient Model-Based Recognition Scheme, Proc. Int'l Conf. Computer Vision, 238249.

[9] Lesk, A.M. (2002) Introduction to bioinformatics, New York : Oxford University Press.

[10] Oyang, Y.-J. and Hwang, S.-C. (2002) An Efficient Learning Algorithm for Function Approximation with Radial Basis Function Networks, In Proceeding of 9th International Conference on Neural Information Processing, 2002.

[11] Oyang, Y.-J., Hwang, S-C, Ou, Y.-Y., Chen, C.-Y., and Chen, Z.-W. (2002) A Novel Learning Algorithm for Data Classification with Radial Basis Function Networks, In Proceedings of 9th International Conference on Neural Information Processing (ICONIP2002), Singapore, 2002.

[12] Silverman, B. W. (1986) Density Estimation for Statistics and Data Analysis, Chapman and Hall, London.

[13] Tu, J.-T. (2003) Protein Active Site Prediction By Matching 3D Structural Data, Master thesis, Department of Computer Science and Information Engineering, National Taiwan University, 2003. 